\title{
A MOBILITY-BASED CHANNEL MODEL FOR WIRELESS SYSTEMS WITH ADAPTIVE MODULATION
}

\section{Miguel Lopez-Guerrero}

\author{
Department of Electrical Engineering \\ Metropolitan Autonomous University \\ Mexico City, Mexico \\ milo@xanum.uam.mx
}

\begin{abstract}
The purpose of this paper is to introduce a model intended to capture the behavior of the wireless channel at time scales which are related to packet transmission times. In this approach it is assumed that the prime factor affecting data rate selection is the path attenuation. We model the wireless channel using a multistate stochastic process where each state corresponds to the nominal data rate achieved by the station when it uses a particular combination of modulation and coding (i.e., adaptive modulation). Differently from other proposals, in the proposed model state changes are created from mobility assumptions. We illustrate the use of this model with a case study.
\end{abstract}

Index Terms- Wireless LAN, random walk mobility model.

\section{INTRODUCTION}

Most of currently used traffic controls for wireless transmissions have been designed using the Gilbert-Elliott channel model $[2,3]$, although more recent investigations (e.g., [1]) suggest that such algorithms may be improved by considering more accurate models. Thus, the popular twostate Gilbert-Elliot model has been extended in several ways. One of such models was introduced in [8] where it was proposed to make use of a multi-state Markov chain in order to model the variations of SNR of the wireless channel. In this proposal each state corresponds to an interval of signalto-noise ratio (SNR). By considering that BPSK is the modulation scheme in use, each state is associated to a nominal bit error rate (BER) and transitions among states are triggered by the Doppler effect. Since Doppler shifts produce fast variations of SNR between the extreme states of the Markov chain, this assumption turns out to be a limiting factor for this model. Even if it is assumed that the SNR remains within a certain interval at least for the time duration of a transmitted symbol, the system should be capable, for example, of encoding and decoding each symbol differently in order to take advantage of this channel model. This assumption is clearly impractical in real communication

\section{Javier Gomez}

\author{
Department of Telecommunications Engineering \\ National Autonomous University of Mexico \\ Mexico City, Mexico \\ javierg@fi-b.unam.mx
}

systems where these changes can only be applied to whole packets, which usually expand several symbols. Nevertheless, based on the model described in [8], other ways of partitioning the range of values for the SNR have also been proposed. For instance, Q. Zhang et al. in [9] proposed a partitioning method intended to achieve about the same number of transmitted packets per state. In [4] the partitioning is done as to analyze the system using a queuing system.

The multi-state channel models described above assume that only one modulation scheme is used for the whole range of SNR values. These models neglect the fact that several modern standards make use of different combinations of modulation and coding according to the present SNR (i.e., adaptive modulation is used to meet performance requirements in terms of BER). For example, by using adaptive modulation, a wireless LAN standard such as IEEE $802.11 \mathrm{~g}$ is capable of transmitting from $1 \mathrm{Mbps}$ for long distances to $54 \mathrm{Mbps}$ for short distances. Similar schemes are found in $3 \mathrm{G}$ systems such as UMTS, $4 \mathrm{G}$ standards such as WiMAX and even PAN networks such as Bluetooth.

In this paper we introduce a model which is intended to represent the wireless channel as seen by a roaming station. With respect to previous proposals found in the literature we also make use of a multistate channel model but, what is different in this work is that a) we are assuming that the wireless communications make use of adaptive modulation and b) state changes are triggered by position changes. In our approach, the time between state changes is large enough as to accommodate one or more packet transmissions.

The remaining of this paper is organized as follows. In Section 2 we describe how the states of the model are created and also how mobility is taken into consideration in order to produce state changes. In our description of the model we initially consider a roaming station serviced by a single base station but we also explain how to consider scenarios with multiple base stations and various users. In Section 3 we present a case study in the context of a currently popular wireless LAN standard. Our purpose in this section is to illustrate how the model can be used to study and evaluate a wireless system. We end this paper in Section 4 with some conclusions and comments. 


\section{THE WIRELESS CHANNEL MODEL}

\subsection{Modeling coverage regions}

Consider an infrastructure-based wireless network. Let us assume that a mobile station, roaming in the service area, is able to monitor the state of its wireless link to the base station. This is not an unrealistic assumption since there are some mechanisms that have already been proposed to accomplish this task. Such techniques include: SNR measurements, sampling of the channel state using the ACK packets returned from the receiver, statistics related to packet losses, probe packets, etc.

As the mobile station roams in the coverage area of the wireless network, communications with the base station are affected by several factors such as position changes, moving speed, multi-path signals, etc. From all these factors we will consider that the main factor affecting achievable transmit data rate is the path attenuation. This is the case where rate adaptation is done according to the observed mean SNR. We also assume that SNR measurements taken by a mobile station decrease as it increases its distance from the base station. This is usually the case in low-mobility applications such as pedestrian users roaming in a wireless LAN. However, this assumption may not hold in other cases, such as in CDMA cellular networks, where open or closed-loop power control is exerted in order to maintain a constant received power at both ends.

In adaptive-modulation systems the full range of SNR is split into several intervals where each one of them is associated with a particular scheme of modulation and coding. Thus, each range of SNR can be associated to a nominal data transmission rate. There is no a general way to make this association, for a given standard each vendor makes the association between SNR and transmission rate.

In our model the service area is divided into several regions, where each region consists of all points that, because of their similar distance to the base station, can be associated to the same data rate. This way, the service area consists of regions of low data rate surrounding areas of higher data rate. We illustrate this situation in Fig. 1 where the base station is assumed to be located in the center and darker areas represent higher rate regions. As depicted in Fig. 1, let us initially assume that the concentric areas are circular. Let us number the rings of the service area in such a way as to start with 1 at the innermost region. The ring number increases as one moves away from the base station. Region $K-1$ corresponds to the outermost ring with coverage and a transition from ring $K-1$ to area $K$ means that a mobile has left the service area. As it is illustrated in Fig. 1, we will denote with $r_{i}$ the radius of the circular boundary between adjacent regions $i$ and $i+1$.

In what follows we describe the proposed model for the coverage regions. First we consider the single-cell network, then the multi-cell case and finally we explain how the model can be generalized as to consider more than one roaming station.

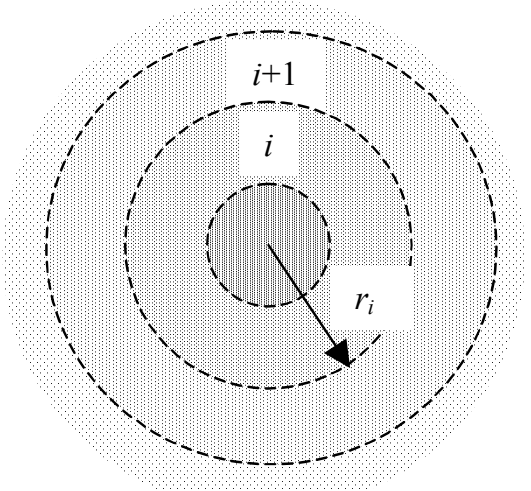

Fig. 1. Service area with regions of different signal strength.

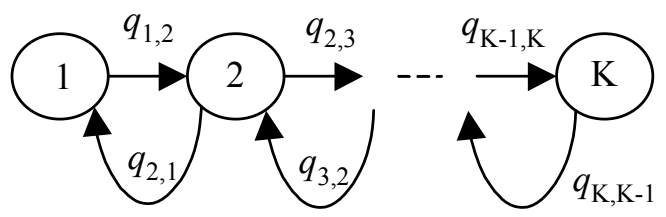

Fig. 2. Continuous-time model for a single cell

\subsection{Single-cell model}

Let us assume that there is always one roaming station so that if it leaves the service area, a new one enters. The mobile station uses adaptive modulation so that it can transmit at different data rates as it changes its distance to the base station.

In order to represent the station roaming in the service area we use a continuous-time stochastic process with state space $\{1, \ldots, K\}$ as depicted in Fig. 2. Let us denote with $Q$ the corresponding matrix of transition rates with elements $\left\{q_{i, j}: i, j \in\{1, \ldots, K\}\right\}$. Differently from other proposals, in this work we determine the elements of this matrix based on a mobility pattern. We will make use of the random walk mobility model, but the basic idea can be explored with other models.

The random walk mobility model is widely used in mobility-related studies. It has been shown (e.g., see [6]) that this model is accurate enough to be used in many scenarios, especially when the mobiles roam following a path which can be considered as lattice like. In this model, a mobile terminal has mean velocity $V$ and, each time it reaches an intersection of the lattice, it picks up a direction of movement uniformly distributed over $[0,2 \pi]$. As explained in [5] (see also Appendix A), for the random walk model the mean cell cross over rate $q$ is given by

$$
q=\frac{V L}{\pi S},
$$


where the cell under consideration has area $S$ and perimeter $L$ (such cell can have an arbitrary form). For a circular cell of radius $r$ the mean cell cross over rate turns out to be

$$
q=\frac{2 \mathrm{~V}}{\pi r}
$$

and the mean cell dwell time $\tau$ can be estimated from the cell cross over rate as

$$
\tau=\frac{1}{q}
$$

From Eq. (2) we can compute the transition rates between the regions of the coverage area (see Fig. 1) as follows

$$
q_{i, j}=q_{j, i}= \begin{cases}(2 V) /\left(\pi r_{i}\right) & \text { if } j=i+1 \\ 0 & \text { if } j>i+1\end{cases}
$$

for $i$ from 1 to $K-1$. In (4) it was taken into consideration that for the system to be in statistical equilibrium the crossing rate out of a region equals the crossing rate into it. The remaining elements $q_{i i}$ can be computed by taking into consideration that for a given $i$

$$
\sum_{j} q_{i, j}=0
$$

From (3) the mean residence time in region $i$ is

$$
\tau_{i}=\frac{1}{q_{i, i+1}}-\frac{1}{q_{i-1, i}} ; \text { where } i=2, \ldots, K-1
$$

and $\tau_{1}$ can be computed directly from (3).

Let us now consider a steady state probability row vector $\Pi$ with elements $\left\{\pi_{k}: k \in\{1, \ldots, K-1\}\right\}$. Each element $\pi_{k}$ indicates the fraction of time that, in the long term, the mobile spends in region $k$. One way to compute these probabilities is to normalize the total coverage area of the cell to one, and then obtain the fraction contributed by each region.

\subsection{Multi-cell network model}

The previously described model applies in a case where the coverage area is served by a single base station. However, a network spanning across a large area needs to be served by several base stations. In this section we show how the model can be adapted to this case.

Consider a multi-cell network as the one illustrated in Fig. 3 where usual hexagon-shape cells are considered. Each cell corresponds to the coverage area of a base station. For convenience we divide each cell into several concentric regions of hexagonal shape. For simplicity, we are illustrating just a few of these regions in two cells of Fig. 3 (bottom right).

As a mobile station roams in the network, it may stay in the same cell or may cross the boundary between two adjacent cells. As also illustrated in Fig. 3, a roaming

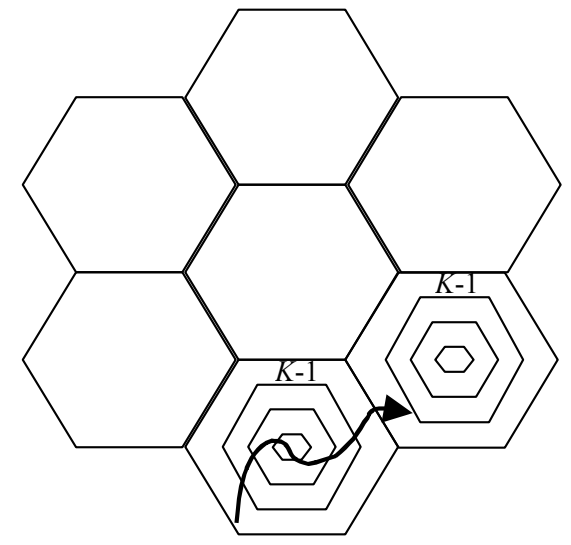

Fig. 3. A multi-cell network.

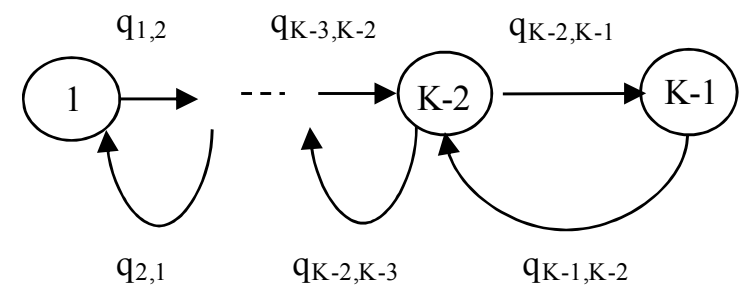

Fig. 4. Continuous-time model for the multi-cell network. It is shown how the two states $K-1$ of adjacent cells are collapsed into a single state $K-1$

terminal performing a handover will move from region $K-1$ in the old cell to region $K-1$ in the new cell. We can consider that each cell can be represented by a multistate model with state space $\{1, \ldots, K-1\}$ and a handover implies a transition between states $K-1$ belonging to two different cells. If the handover rate is not a relevant issue in the evaluation of the system and assuming that all cells have the same characteristics (i.e., user density, mobility patterns and radio interface), the two states $K-1$ representing two adjacent regions of identical data rates can be represented with an equivalent state as shown in Fig. 4.

Note that transition rates appearing in this model are different from the ones previously obtained for a circular cell since now we are assuming that the cells are hexagons and therefore, regions with different data rates are assumed to be also hexagonal. Nevertheless, these transition rates can be easily computed from (1) in a similar way as we did before for circular regions. Let us again assume that the mobile moves according to the random walk mobility model. With this information the cross over rate out of a hexagonal area can be obtained. Recall that the area and perimeter of a hexagon are given by $S=(3 \sqrt{3} / 2) r^{2}$ and $L=6 r$ respectively, where $r$ is the circumradius (distance measured from the center to one vertex). Therefore, in this case (1) becomes: 


$$
q=\frac{4 V}{\sqrt{3} \pi r} .
$$

Thus, the transition rates between hexagonal regions of the model can be computed as:

$$
q_{i, j}=q_{j, i}= \begin{cases}(4 V) /\left(\sqrt{3} \pi r_{i}\right) & \text { if } j=i+1, \\ 0 & \text { if } j>i+1 .\end{cases}
$$

for $i$ from 1 to $K-2$. Remaining elements $q_{i i}$ are computed as indicated by (5).

Steady state probabilities can be obtained using geometric arguments as we did in the single-cell case. In the present case it has to be considered that the area represented by state $K-1$ has contributions from adjacent cells. We propose to consider a virtual hexagonal cell where the area of its outermost region represents the coverage achieved by adjacent regions in the multi-cell model. In order to define such polygon we proceed as follows. Let $S_{i}$ represent the area of a hexagon of circumradius $r_{i}$. Because region $K-1$ in the virtual cell represents the coverage area created by the outermost regions of two adjacent cells, it must be given by $2\left(S_{K-1}-S_{K-2}\right)$. In the virtual cell, this area surrounds a hexagon of area $S_{K-2}$ so that the total area of the virtual cell $\hat{S}_{K-1}$ can be found as $2\left(S_{K-1}-S_{K-2}\right)+S_{K-2}$, after some algebra:

$$
\hat{S}_{K-1}=\frac{3 \sqrt{3}}{2}\left(2 r_{K-1}^{2}-r_{K-2}^{2}\right) .
$$

From (9) it can be inferred that the hexagon $K-1$ in the virtual hexagonal cell has a virtual circumradius given by $\hat{r}_{K-1}=\sqrt{2 r_{K-1}^{2}-r_{K-2}^{2}}$. Thus, in order to find the steady state probabilities the total area of the virtual cell has to be normalized to one and then the fraction contributed by each region can be computed.

We end this section with a few comments that are worth mentioning. It may appear as tantalizing to try to apply the theory of Markov processes to the analysis of the previously described system. Indeed, if we study this system when state changes take place, we can obtain an embedded Markov chain. However, we cannot obtain a Markov chain from the well known method of sampling of the matrix of transition rates at arbitrary points in time. This is due to the fact that there is no evidence that the random walk mobility model leads to sojourn times that are exponentially distributed, as required by the Markov theory.

\subsection{Multi-terminal model}

So far we have considered in our analysis a single mobile station. However, we can easily extend our model in order to consider multiple stations roaming in the network and thus, obtain system wide statistics such as overall cross over rates.
Assume that each mobile station roams according to the random walk mobility model. With this assumption in place they remain evenly distributed and maintain a constant density if a station that leaves is replaced by another one that enters the service area. We can compute the overall crossover rates for a given area as [6]

$$
q=\frac{\rho V L}{\pi}
$$

where $\rho$ is the mobile density, $V$ is the mean velocity and $L$ is the perimeter of the area being considered. For instance, for a circular cell of radius $r$ the cell crossover rate is

$$
q=2 \rho V r \text {. }
$$

Thus, in our single-cell circular network model the transition rates between adjacent regions become

$$
q_{i, j}=q_{j, i}=\left\{\begin{array}{l}
2 \rho V r_{i} \quad \text { if } j=i+1 \\
0 \quad \text { if } j>i+1
\end{array}\right.
$$

and remaining transition rates can be determined from (5).

Statistics regarding overall transition rates can be useful, for instance, to determine the required processing speed of a central controller in a system where the rate of location changes becomes an important issue.

It is worth mentioning that one can compute individual crossover rates (i.e., Eq. (1)) from total transition rates (i.e., Eq. (10)) and vice versa. This transformation basically consists in the scaling of one or the other considering the number of terminals present in the area.

\section{CASE STUDY: WIFI IEEE 802.11G}

We illustrate some of the ideas of this paper with a case study based on IEEE $802.11 \mathrm{~g}$ since it is an example of a currently popular adaptive modulation wireless system. Here is a summary of our findings.

\subsection{Experimental map of a service area}

In the first set of experiments our aim was to determine how realistic our assumption regarding the shape of the coverage regions is. We conducted a series of measurements on an open area located in the National Autonomous University of Mexico main campus. This is an example of typical school premises for outdoor study and leisure activities. There was only one access point and measurements were obtained positioning a laptop equipped with a Wi-Fi network card at various places. The results are depicted in Fig. 5 where the access point was located at the point labeled as "AP" and the numbers indicate the measured data rate in Mbps. As also depicted in the figure, the regions of different data rates resulted concentric and roughly circular. It is important to mention that this situation may not happen in indoor scenarios due to the fact the building layout creates coverage regions of capricious shapes. 


\subsection{Use of the model in performance evaluation}

In the second set of experiment our aim is to illustrate how the model can be used to represent the service provided by a wireless network and quickly determine some metrics of performance.

We start by considering available data regarding data rate vs. range which are usually provided by vendors. An example of this is shown in Table I. Let us consider an $802.11 \mathrm{~g}$ access point of such characteristics and a mobile

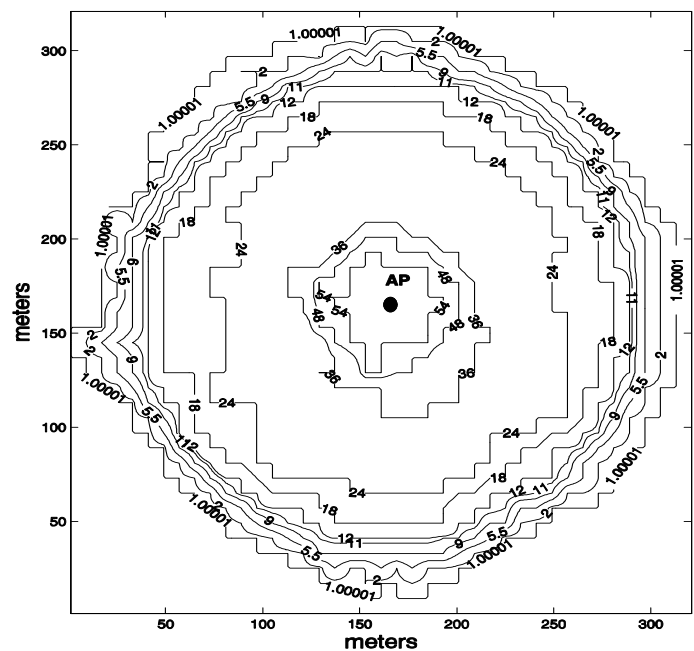

station that roams in its service area. The mobile user roams with a mean speed of $1 \mathrm{~m} / \mathrm{s}$ (i.e., pedestrian speed) and following a random walk mobility pattern. We assumed circular coverage regions and we computed the mean transition rates from (4). From these data we also computed the mean sojourn time in each region. All these results are shown in Table II. Under the same assumptions, we tested these results with computer simulations (100 simulations of $10 \times 10^{6}$ time steps each) and for all practical purposes the results were the same.

From Table I we also computed the steady state probabilities (i.e., fraction of time that in the long-term the user spends in each region). These results are shown in Table III. From these probabilities the mean data rate can be easily computed, it turns out that the roaming station gets on average around $10 \mathrm{Mbps}$.

\section{CONCLUSION}

We underlined the basics of a channel model based on mobility patterns. The proposed model can be used to analyze some low-mobility scenarios such as pedestrian users roaming in an outdoors WLAN. The model can be made more complex by considering other mobility patters or different shapes for the service area. We believe that a mobility-based channel model is a useful approach to quickly estimate some aspects of the performance of modern wireless systems.

Fig. 5 Regions with different data rates created in an 802.11g WLAN

TABLE I. IEEE 802.11g EXPECTED DATA RATES AND OTHER CHARACTERISTICS, BASED ON DATA FROM REF. [7]

\begin{tabular}{ccccccccccc}
\hline Data rate $(\mathrm{Mbps})$ & 54 & 48 & 36 & 24 & 18 & 12 & 9 & 6 & 2 & 1 \\
\hline Transmission type & OFDM & OFDM & OFDM & OFDM & OFDM & OFDM & OFDM & OFDM & DSSS & DSSS \\
\hline Modulation & 64QAM & 64QAM & 16QAM & 16QAM & QPSK & QPSK & BPSK & BPSK & QPSK & BPSK \\
\hline $\begin{array}{c}\text { Max. distance } \\
\text { from the AP }(\mathrm{m})\end{array}$ & 19 & 23 & 35 & 42 & 50 & 54 & 57 & 65 & 77 & 100 \\
\hline
\end{tabular}

TABLE II. MEAN TRANSITION RATES AND MEAN SOJOURN TIMES FOR A CASE STUDY WITH A MOBILE SPEED OF $1(\mathrm{~m} / \mathrm{s})$

\begin{tabular}{|c|c|c|c|c|c|c|c|c|c|c|}
\hline Radius $(\mathrm{m}) *$ & 19 & 23 & 35 & 42 & 50 & 54 & 57 & 65 & 77 & 100 \\
\hline Crossings/s & 0.0335 & 0.0277 & 0.0182 & 0.0152 & 0.0127 & 0.0118 & 0.0112 & 0.0098 & 0.0083 & 0.0064 \\
\hline Sojourn t.(s) & 29.85 & 6.25 & 18.84 & 10.85 & 12.95 & 6.01 & 4.54 & 12.76 & 18.44 & 35.77 \\
\hline
\end{tabular}

TABLE III. STEADY STATE PROBABILITIES

\begin{tabular}{|c|c|c|c|c|c|c|c|c|c|c|}
\hline State & 1 & 2 & 3 & 4 & 5 & 6 & 7 & 8 & 9 & 10 \\
\hline $\begin{array}{c}\text { Data rate } \\
\text { (Mbps) }\end{array}$ & 54 & 48 & 36 & 24 & 18 & 12 & 9 & 6 & 2 & 1 \\
\hline $\begin{array}{l}\text { Steady state } \\
\text { probability }\end{array}$ & 0.0361 & 0.0168 & 0.0696 & 0.0539 & 0.0736 & 0.0416 & 0.0333 & 0.0976 & 0.1704 & 0.4071 \\
\hline
\end{tabular}




\section{APPENDIX A. Mean cell cross over rates.}

The main formulae used in this work are derived in this appendix since the authors could not find a suitable reference.

Let us assume a discrete version of the random walk mobility pattern as it would be implemented in a discrete event simulator. At the beginning of the simulation the mobile stations are positioned at random across the simulation area, thus creating a uniform density $\rho$. Each station randomly picks up a moving direction between 0 and $2 \pi$ and moves in that direction at a constant speed of $V$ for a time step of duration $\Delta$. Upon reaching the destination, a new moving direction is randomly selected and the process is repeated again. If a mobile station crosses the outer boundary of the simulation area, a new station is generated in a mirrored position. Consider a simple closed curve with no singular points enclosing part of this simulation scenario. We illustrate this situation with area $S$ in Figure A1 (bottom left). Let us compute the mean cell cross over rates, i.e., the expected number of users that move out of $S$ per time time slot.

Observe that in one time step each station will have a displacement of $V \Delta$. Let us assume that the size and shape of $S$ is such that the curvature radius of its boundary is significantly larger than $V \Delta$. Under this assumption there is a region close to the border of thickness $V \Delta$ where the stations that may be able to leave $S$ are (the inner boundary of this region in illustrated in Fig. A1 with a dashed line). Consider one station in this strip and let $x$ be its shortest distance to the border (this direction can be found by noting that it should be perpendicular to the tangential vector at the crossing point). Initially assume that $x$ is known. As depicted in Figure A1 if the station moves at an angle which is less than $\theta$, it leaves area $S$. Note that $x, V \Delta$ and $\theta$ satisfy the relation $\cos \theta=x /(V \Delta)$ and therefore $\theta=\cos ^{-1}(x /(V \Delta))$. Thus, given that $x$ is known and the curvature radius is aasumed to be so large that it negligibly changes in the neighborhood of the crossing point ( $V \Delta$ is small), the probability that the station leaves the area in one time slot is

$$
P[\text { out } \mid x]=\frac{2 \theta}{2 \pi}=\frac{\cos ^{-1}\left(\frac{x}{V \Delta}\right)}{\pi} .
$$

The dependence on $x$ can be removed by taking into consideration that since the station was placed at random then $x \sim U[0, V \Delta]$ and therefore,

$$
P_{\text {out }}=\int_{0}^{V \Delta} P[\text { out } \mid x] f_{X}(x) d x=\int_{0}^{V \Delta} \frac{\cos ^{-1}\left(\frac{x}{V \Delta}\right)}{\pi} \frac{1}{V \Delta} d x .
$$

Integrating by parts we obtain

$$
P_{\text {out }}=\frac{1}{\pi} \text {. }
$$

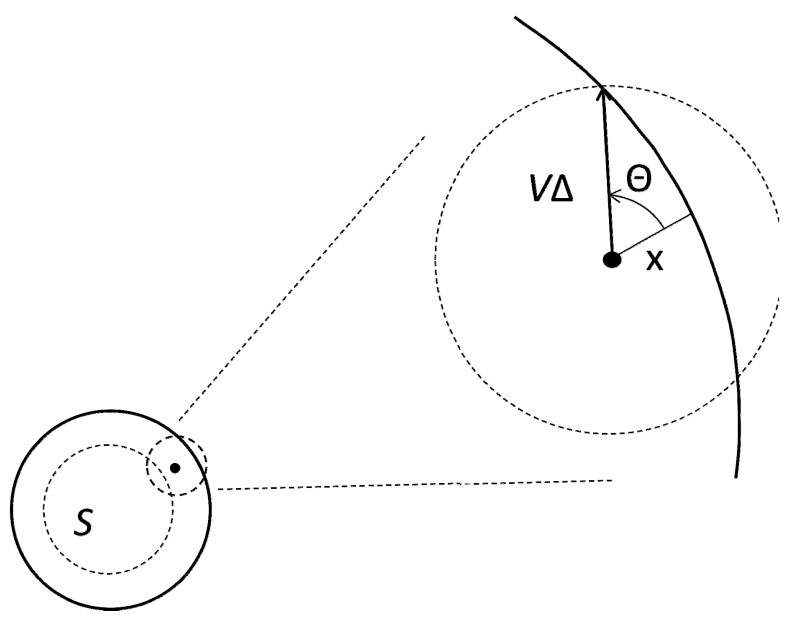

Fig. A1. Representation of a coverage area and a roaming station.

The overall mean cross over rate can be obtained by considering all the stations that are located in the strip. Recalling again that the curvature radius is large and $V \Delta$ is small, the area of the strip can be approximated and thus

$$
q_{\text {all }}=\left(L V \Delta \rho P_{\text {out }}\right) \frac{1}{\Delta}=\rho \frac{V L}{\pi}
$$

where $L$ is the perimeter of area $S$. If we divide by the total number of users in $S$ we obtain the mean cross over rate for a single station

$$
q_{\text {one }}=\frac{q_{\text {all }}}{\text { stations }}=\rho \frac{V L}{\pi} \frac{1}{\rho S}=\frac{V L}{\pi S} .
$$

\section{REFERENCES}

[1] A. Azgin and M. Krunz, "Impact of channel modeling on the performance of wireless scheduling schemes," In Proceedings of 2003 IEEE Vehicular Technology Conference (VTC 2003), October 4-9, 2003.

[2] E. O. Elliott, "Estimates for codes on burst-noise channels," The Bell System Technical Journal, vol. 42, pp. 1977-1997, September 1963.

[3] E. N. Gilbert, "Capacity of a burst-noise channel," The Bell System Technical Journal, vol. 39, pp. 1253-1265, September 1960.

[4] M. Hassan and M. M. Krunz, "Markov-based channel characterization for tractable performance analysis in wireless packet networks," IEEE Transactions in Wireless Communications, vol. 3, no. 3, 2004.

[5] B. Jabbari, "Teletraffic aspects of evolving and next-generation networks," IEEE Personal Communications, vol. 3, no. 6, pp. 4-9, December 1996.

[6] I. Seskar, S. V. Marić, J. Holtzman and J. Wasserman, Rate of location updates in cellular systems, in Proc. of Vehicular Technology Conference 1992.

[7] Broadcom Corporation, "IEEE 802.11g: The new mainstream wireless LAN standard", white paper, document 802.11g-WP104-R. [Online: www.54g.org] Retrieved June 2006.

[8] H. S. Wang and N. Moayeri, "Finite-state Markov channel -a useful model for radio communication channels," IEEE Transactions on Vehicular Technology, vol. 4, no. 1, February 1995.

[9] Q. Zhang and S. A. Kassam, "Finite-state Markov model for Rayleigh fading channels," IEEE Transactions on Communications, vol. 47, no. 11, pp. 1688-1692, November 1999 